\title{
A STUDY OF HARMONIC SECTIONS OF TANGENT BUNDLES WITH VERTICALLY RESCALED BERGER-TYPE DEFORMED SASAKI METRIC
}

\author{
ABDERRAHIM ZAGANE
}

\begin{abstract}
In this paper, we introduce a vertically rescaled Bergertype deformed Sasaki metric on the tangent bundle $T M$ over an antiparaKähler manifold $(M, \varphi, g)$. We study the harmonicity of the tangent bundle equipped with the vertically rescaled Berger-type deformed Sasaki metric and we establish a necessary and sufficient condition under which a vector field is harmonic with respect to this metric. We also construct some examples of harmonic vector fields. Finally, we study the harmonicity of a vector field along a map between Riemannian manifolds, the target manifold being anti-paraKähler equipped with a vertically rescaled Berger-type deformed Sasaki metric on its tangent bundle. Also, we discuss the harmonicity of the composition of the projection map of the tangent bundle of a Riemannina manifold with a map from this manifold into another Riemannian manifold, the source manifold being anti-paraKähler whose tangent bundle is endowed with a vertically rescaled Berger-type deformed Sasaki metric.
\end{abstract}

\section{Introduction}

The geometry of the tangent bundle $T M$ over a Riemannian manifold $(M, g)$, equipped with the Sasaki metric has been studied by many authors such as S. Sasaki [16], P. Dombrowski [5], K. Yano, S. Ishihara [19], A. Salimov, A. Gezer, K. Akbulut [13] etc. The rigidity of Sasaki metric has incited some geometers to construct and study other metrics on $T M$. E. Musso, F. Tricerri has introduced the notion of Cheeger-Gromoll metric [11], this metric has been studied also by many authors (see $[8,15,17]$ ). M. Altunbas, R. Simsek, A. Gezer has introduced the notion of Berger type deformed Sasaki metric [2].

Consider a smooth map $\phi:\left(M^{m}, g\right) \rightarrow\left(N^{n}, h\right)$ between two Riemannian manifolds, then the second fundamental form of $\phi$ is defined by

$$
(\nabla d \phi)(X, Y)=\nabla_{X}^{\phi} d \phi(Y)-d \phi\left(\nabla_{X} Y\right)
$$

2010 Mathematics Subject Classification. 53C43, 58E20, 53C20, 53C55.

Key words and phrases. Tangent bundle, vertically rescaled Berger-type deformed Sasaki metric, paracomplex structure, harmonic maps. 
Here $\nabla$ is the Riemannian connection on $M$ and $\nabla^{\phi}$ is the pull-back connection on the pull-back bundle $\phi^{-1} T N$, and

$$
\tau(\phi)=\operatorname{trace}_{g} \nabla d \phi
$$

is the tension field of $\phi$.

The energy functional of $\phi$ is defined by

$$
E(\phi)=\int_{K} e(\phi) d v_{g}
$$

such that $K$ is any compact of $M$, where

$$
e(\phi)=\frac{1}{2} \operatorname{trace}_{g} h(d \phi, d \phi)
$$

is the energy density of $\phi$.

A map is called harmonic if it is a critical point of the energy functional E. For any smooth variation $\left\{\phi_{t}\right\}_{t \in I}$ of $\phi$ with $\phi_{0}=\phi$ and $V=\left.\frac{d}{d t} \phi_{t}\right|_{t=0}$, we have

$$
\left.\frac{d}{d t} E\left(\phi_{t}\right)\right|_{t=0}=-\int_{K} h(\tau(\phi), V) d v_{g}
$$

Then $\phi$ is harmonic if and only if $\tau(\phi)=0$.

One can refer to $[6,7,9,12]$ for background on harmonic maps.

The main idea in this note consists in the deformation (in the vertical bundle) of the Berger type deformed Sasaki metric on the tangent bundle [2]. Firstly, we introduce the vertically rescaled Berger-type deformed Sasaki metric on the tangent bundle $T M$ over an anti-paraKähler manifold $\left(M^{2 m}, \varphi, g\right)$ and we investigate the Levi-Civita connection (Theorem 3.1). Secondly, we study the harmonicity respect to the vertically rescaled Berger-type deformed Sasaki metric and we establish necessary and sufficient conditions under which a vector field is harmonic (Theorem 4.2 and Theorem 4.3). We also construct some examples of harmonic vector fields (Example 4.1 and Example 4.2). After that we study the harmonicity of the map $\sigma:(M, g) \longrightarrow)(T N, \tilde{h}), x \rightarrow(\phi(x), v)$ (Theorem 4.4 and Theorem $4.5)$ and the map $\Phi:(T M, \tilde{g}) \longrightarrow(N, h),(x, u) \rightarrow \phi(x) \quad$ ( Theorem 4.6 and Theorem 4.7), where $\phi:(M, g) \longrightarrow(N, h)$ is a smooth map and $(T N, \tilde{h})$ (resp $(T M, \tilde{g}))$ is a tangent bundle equipped with the vertically rescaled Berger-type deformed Sasaki metric on $N(\operatorname{resp}$ on $M)$.

\section{Preliminaries}

Let $T M$ be the tangent bundle over an $m$-dimensional Riemannian manifold $\left(M^{m}, g\right)$ and the natural projection $\pi: T M \rightarrow M$. A local chart $\left(U, x^{i}\right)_{i=\overline{1, m}}$ on $M$ induces a local chart $\left(\pi^{-1}(U), x^{i}, y^{i}\right)_{i=\overline{1, m}}$ on $T M$. Denote by $\Gamma_{i j}^{k}$ the Christoffel symbols of $g$ and by $\nabla$ the Levi-Civita connection of $g$. Let $C^{\infty}(M)$ be the ring of real-valued $C^{\infty}$ functions on $M$ and $\Im_{0}^{1}(M)$ be the module over $C^{\infty}(M)$ of $C^{\infty}$ vector fields on $M$.

The Levi Civita connection $\nabla$ defines a direct sum decomposition

$$
T_{(x, u)} T M=V_{(x, u)} T M \oplus H_{(x, u)} T M .
$$


of the tangent bundle to $T M$ at any $(x, u) \in T M$ into vertical subspace

$$
V_{(x, u)} T M=\operatorname{Ker}\left(d \pi_{(x, u)}\right)=\left\{\left.\xi^{i} \frac{\partial}{\partial y^{i}}\right|_{(x, u)}, \xi^{i} \in \mathbb{R}\right\},
$$

and the horizontal subspace

$$
H_{(x, u)} T M=\left\{\left.\xi^{i} \frac{\partial}{\partial x^{i}}\right|_{(x, u)}-\left.\xi^{i} u^{j} \Gamma_{i j}^{k} \frac{\partial}{\partial y^{k}}\right|_{(x, u)}, \xi^{i} \in \mathbb{R}\right\} .
$$

Note that the map $X \rightarrow X^{H}$ is an isomorphism between the vector spaces $T_{x} M$ and $H_{(x, u)} T M$. Similarly, the map $X \rightarrow X^{V}$ is an isomorphism between the vector spaces $T_{x} M$ and $V_{(x, u)} T M$. Obviously, each tangent vector $Z \in T_{(x, u)} T M$ can be written in the form $Z=X^{H}+Y^{V}$, where $X, Y \in T_{x} M$ are uniquely determined vectors.

Let $X=X^{i} \frac{\partial}{\partial x^{i}}$ be a local vector field on $M$. The vertical and the horizontal lifts of $X$ are defined by

$$
\begin{aligned}
X^{V} & =X^{i} \frac{\partial}{\partial y^{i}} \\
X^{H} & =X^{i} \frac{\delta}{\delta x^{i}}=X^{i}\left\{\frac{\partial}{\partial x^{i}}-y^{j} \Gamma_{i j}^{k} \frac{\partial}{\partial y^{k}}\right\} .
\end{aligned}
$$

For consequences, we have $\left(\frac{\partial}{\partial x^{i}}\right)^{H}=\frac{\delta}{\delta x^{i}}$ and $\left(\frac{\partial}{\partial x^{i}}\right)^{V}=\frac{\partial}{\partial y^{i}}$, then $\left(\frac{\delta}{\delta x^{i}}, \frac{\partial}{\partial y^{i}}\right)_{i=1, m}$ is a local adapted frame on $T T M$.

If $U$ be a local vector field constant on each fiber $T_{x} M$ i.e. $\left(U=u=u^{i} \frac{\partial}{\partial x^{i}}\right)$, the vertical lift $U^{V}$ is called the canonical vertical vector field or Liouville vector field on $T M$.

Lemma 2.1. $[5,19]$ Let $(M, g)$ be a Riemannian manifold. The bracket operation of vertical and horizontal vector fields is given by the formulas

(1) $\left[X^{H}, Y^{H}\right]_{(x, u)}=[X, Y]_{(x, u)}^{H}-\left(R_{x}(X, Y) u\right)^{V}$,

(2) $\left[X^{H}, Y^{V}\right]_{(x, u)}=\left(\nabla_{X} Y\right)_{(x, u)}^{V}$,

(3) $\left[X^{V}, Y^{V}\right]_{(x, u)}=0$,

for all vector fields $X, Y \in \Im_{0}^{1}(M)$ and $(x, u) \in T M$, where $\nabla$ and $R$ denotes respectively the Levi-Civita connection and the curvature tensor of $(M, g)$.

\section{Vertically rescaled Berger-type deformed Sasaki metric}

Let $M$ be a $2 m$-dimensional Riemannian manifold with a Riemannian metric g. An almost paracomplex manifold is an almost product manifold $\left(M^{2 m}, \varphi\right)$, $\varphi^{2}=i d$, such that the two eigenbundles $T^{+} M$ and $T^{-} M$ associated to the two eigenvalues +1 and -1 of $\varphi$, respectively, have the same rank.

A paracomplex structure is an integrable almost paracomplex structure. Let $\left(M^{2 m}, \varphi\right)$ be an almost paracomplex manifold. A Riemannian metric $g$ is said to be an anti-paraHermitian metric if

$$
g(\varphi X, \varphi Y)=g(X, Y)
$$

or equivalently (purity condition), (B-metric)[14]

$$
g(\varphi X, Y)=g(X, \varphi Y)
$$


for all $X, Y \in \Im_{0}^{1}(M)$.

If $\left(M^{2 m}, \varphi\right)$ is an almost paracomplex manifold with an anti-paraHermitian metric $g$, then the triple $\left(M^{2 m}, \varphi, g\right)$ is said to be an almost anti-paraHermitian manifold (an almost B-manifold)[14]. Moreover, $\left(M^{2 m}, \varphi, g\right)$ is said to be antiparaKähler manifold (B-manifold)[14] if $\varphi$ is parallel with respect to the LeviCivita connection $\nabla$ of $g$ i.e. $(\nabla \varphi=0)$.

As is well known, the anti-paraKähler condition $(\nabla \varphi=0)$ is equivalent to paraholomorphicity of the anti-paraHermitian metric $g$, that is, $\left(\phi_{\varphi} g\right)=0$, where $\phi_{\varphi}$ is the Tachibana operator [18].

It is well known that if $\left(M^{2 m}, \varphi, g\right)$ is a anti-paraKähler manifold, the Riemannian curvature tensor is pure [14], and

$$
\begin{cases}R(\varphi Y, Z) & =R(Y, \varphi Z)=R(Y, Z) \varphi=\varphi R(Y, Z), \\ R(\varphi Y, \varphi Z) & =R(Y, Z),\end{cases}
$$

for all $Y, Z \in \Im_{0}^{1}(M)$.

Definition 3.1. Let $\left(M^{2 m}, \varphi, g\right)$ be an almost anti-paraHermitian manifold and $f: M \rightarrow] 0,+\infty[$ be a strictly positive smooth function on $M$. Define a fiber-wise vertically rescaled Berger-type deformed Sasaki metric noted $\tilde{g}$ on $T M$, by

$$
\begin{aligned}
& \tilde{g}\left(X^{H}, Y^{H}\right)=g(X, Y), \\
& \tilde{g}\left(X^{H}, Y^{V}\right)=0, \\
& \tilde{g}\left(X^{V}, Y^{V}\right)=f\left(g(X, Y)+\delta^{2} g(X, \varphi u) g(Y, \varphi u)\right),
\end{aligned}
$$

for all $X, Y \in \Im_{0}^{1}(M)$, where $\delta$ is some constant [2] and $f$ is called twisting function.

Remark 3.1. 1. If $f=1$ and $\delta=0, \tilde{g}$ is the Sasaki metric[16],

2. If $f=1, \tilde{g}$ is the Berger-type deformed Sasaki metric[2],

3. If $\delta=0, \tilde{g}$ is the vertical rescaled metric [4],

4. $\tilde{g}\left(X^{V}, \varphi U^{V}\right)=\left(1+\delta^{2} r^{2}\right) f g(X, \varphi u)$ and $r^{2}=g(u, u)$, for any $X \in \Im_{0}^{1}(M)$.

In the following, we consider $\lambda=1+\delta^{2} r^{2}$ and $r^{2}=g(u, u)=\|u\|^{2}$, where $\|$. denote the norm with respect to $(M, g)$.

Lemma 3.1. [1] Let $(M, g)$ be a Riemannian manifold and $\rho: \mathbb{R} \rightarrow \mathbb{R}$ a smooth function. Then we have

(1) $X^{H}\left(\rho\left(r^{2}\right)\right)=0$,

(2) $X^{V}\left(\rho\left(r^{2}\right)\right)=2 \rho^{\prime}\left(r^{2}\right) g(X, u)$,

(3) $X^{H} g(Y, u)=g\left(\nabla_{X} Y, u\right)$,

(4) $X^{V} g(Y, u)=g(X, Y)$,

for any $X, Y \in \Im_{0}^{1}(M)$.

Lemma 3.2. Let $\left(M^{2 m}, \varphi, g\right)$ be an anti-paraKähler manifold, we have the following:

(1) $X^{H} g(Y, \varphi u)=g\left(\nabla_{X} Y, \varphi u\right)$,

(2) $X^{V} g(Y, \varphi u)=g(Y, \varphi X)$,

for all $X, Y \in \Im_{0}^{1}(M)$.

Proof. The results follow immediately from Lemma 3.1. 
Lemma 3.3. Let $\left(M^{2 m}, \varphi, g\right)$ be an anti-paraKähler manifold, we have the following:

(1) $X^{H} \tilde{g}\left(Y^{V}, Z^{V}\right)=\frac{1}{f} X(f) \tilde{g}\left(Y^{V}, Z^{V}\right)+\tilde{g}\left(\left(\nabla_{X} Y\right)^{V}, Z^{V}\right)+\tilde{g}\left(Y^{V},\left(\nabla_{X} Z\right)^{V}\right)$,

(2) $X^{V} \tilde{g}\left(Y^{V}, Z^{V}\right)=\delta^{2} f[g(X, \varphi Y) g(Z, \varphi u)+g(Y, \varphi u) g(X, \varphi Z)]$,

where $X, Y, Z \in \Im_{0}^{1}(M)$.

Proof. The results follow directly from Lemma 3.1 and Lemma 3.2

We shall calculate the Levi-Civita connection $\widetilde{\nabla}$ of $T M$ with vertically rescaled Berger-type deformed Sasaki metric $\tilde{g}$. This connection is characterized by the Koszul formula:

$$
\begin{aligned}
2 \tilde{g}\left(\widetilde{\nabla}_{\tilde{X}} \tilde{Y}, \widetilde{Z}\right)= & \tilde{X} \tilde{g}(\tilde{Y}, \widetilde{Z})+\widetilde{Y} \tilde{g}(\widetilde{Z}, \tilde{X})-\widetilde{Z} \tilde{g}(\tilde{X}, \tilde{Y}) \\
& +\tilde{g}(\widetilde{Z},[\tilde{X}, \tilde{Y}])+\tilde{g}(\tilde{Y},[\widetilde{Z}, \widetilde{X}])-\tilde{g}(\tilde{X},[\tilde{Y}, \widetilde{Z}]) .
\end{aligned}
$$

for all $\tilde{X}, \tilde{Y}, \widetilde{Z} \in \Im_{0}^{1}(T M)$.

Theorem 3.1. Let $\left(M^{2 m}, \varphi, g\right)$ be an anti-paraKähler manifold and $(T M, \tilde{g})$ its tangent bundle equipped with the vertically rescaled Berger-type deformed Sasaki metric, then we have the following formulas.

$$
\begin{aligned}
\text { 1. } \widetilde{\nabla}_{X^{H}} Y^{H} & =\left(\nabla_{X} Y\right)^{H}-\frac{1}{2}(R(X, Y) u)^{V}, \\
\text { 2. } \widetilde{\nabla}_{X^{H}} Y^{V} & =\left(\nabla_{X} Y\right)^{V}+\frac{1}{2 f} X(f) Y^{V}+\frac{f}{2}(R(u, Y) X)^{H}, \\
\text { 3. } \widetilde{\nabla}_{X^{V}} Y^{H} & =\frac{1}{2 f} Y(f) X^{V}+\frac{f}{2}(R(u, X) Y)^{H}, \\
\text { 4. } \widetilde{\nabla}_{X^{V}} Y^{V} & =\frac{-1}{2 f} \tilde{g}\left(X^{V}, Y^{V}\right)(\operatorname{grad} f)^{H}+\frac{\delta^{2}}{\lambda} g(X, \varphi Y)(\varphi U)^{V},
\end{aligned}
$$

for all vector fields $X, Y \in \Im_{0}^{1}(M)$, where $\nabla$ and $R$ denotes respectively the LeviCivita connection and the curvature tensor of $\left(M^{2 m}, \varphi, g\right)$.

Proof. The proof of Theorem 3.1 follows directly from Kozul formula (3.4), Lemma 2.1, Lemma 3.2 and Lemma 3.3.

(1) Direct calculations give,

$$
\begin{aligned}
2 \tilde{g}\left(\widetilde{\nabla}_{X^{H}} Y^{H}, Z^{H}\right)= & X^{H} \tilde{g}\left(Y^{H}, Z^{H}\right)+Y^{H} \tilde{g}\left(Z^{H}, X^{H}\right)-Z^{H} \tilde{g}\left(X^{H}, Y^{H}\right) \\
& +\tilde{g}\left(Z^{H},\left[X^{H}, Y^{H}\right]\right)+\tilde{g}\left(Y^{H},\left[Z^{H}, X^{H}\right]\right) \\
& -\tilde{g}\left(X^{H},\left[Y^{H}, Z^{H}\right]\right) \\
= & X g(Y, Z)+Y g(Z, X)-Z g(X, Y)+g(Z,[X, Y]) \\
& +g(Y,[Z, X])-g(X,[Y, Z]) \\
= & 2 g\left(\nabla_{X} Y, Z\right) \\
= & 2 \tilde{g}\left(\left(\nabla_{X} Y\right)^{H}, Z^{H}\right),
\end{aligned}
$$


and

$$
\begin{aligned}
2 \tilde{g}\left(\widetilde{\nabla}_{X^{H}} Y^{H}, Z^{V}\right)= & X^{H} \tilde{g}\left(Y^{H}, Z^{V}\right)+Y^{H} \tilde{g}\left(Z^{V}, X^{H}\right)-Z^{V} \tilde{g}\left(X^{H}, Y^{H}\right) \\
& +\tilde{g}\left(Z^{V},\left[X^{H}, Y^{H}\right]\right)+\tilde{g}\left(Y^{H},\left[Z^{V}, X^{H}\right]\right) \\
& -\tilde{g}\left(X^{H},\left[Y^{H}, Z^{V}\right]\right) \\
= & \tilde{g}\left(Z^{V},\left[X^{H}, Y^{H}\right]\right) \\
= & -\tilde{g}\left((R(X, Y) u)^{V}, Z^{V}\right) .
\end{aligned}
$$

we have:

$$
\widetilde{\nabla}_{X^{H}} Y^{H}=\left(\nabla_{X} Y\right)^{H}-\frac{1}{2}(R(X, Y) u)^{V} .
$$

(2) By straightforward calculations,

$$
\begin{aligned}
2 \tilde{g}\left(\widetilde{\nabla}_{X^{H}} Y^{V}, Z^{H}\right)= & X^{H} \tilde{g}\left(Y^{V}, Z^{H}\right)+Y^{V} \tilde{g}\left(Z^{H}, X^{H}\right)-Z^{H} \tilde{g}\left(X^{H}, Y^{V}\right) \\
& +\tilde{g}\left(Z^{H},\left[X^{H}, Y^{V}\right]\right)+\tilde{g}\left(Y^{V},\left[Z^{H}, X^{H}\right]\right) \\
& -\tilde{g}\left(X^{H},\left[Y^{V}, Z^{H}\right]\right) \\
= & \tilde{g}\left(Y^{V},\left[Z^{H}, X^{H}\right]\right) \\
= & -\tilde{g}\left((R(Z, X) u)^{V}, Y^{V}\right) \\
= & -f\left[g(R(Z, X) u, Y)+\delta^{2} g(Y, \varphi u) g(R(Z, X) u, \varphi u)\right] \\
= & f \tilde{g}\left((R(u, Y) X)^{H}, Z^{H}\right) .
\end{aligned}
$$

Where

$$
-g(R(Z, X) u, Y)=g(R(u, Y) X, Z)=\tilde{g}\left((R(u, Y) X)^{H}, Z^{H}\right),
$$

and from (3.3) we have

$$
g(R(Z, X) u, \varphi u)=g(\varphi R(Z, X) u, u)=g(R(\varphi Z, X) u, u)=0 .
$$

It follows from.

$$
\begin{aligned}
2 \tilde{g}\left(\widetilde{\nabla}_{X^{H}} Y^{V}, Z^{V}\right)= & X^{H} \tilde{g}\left(Y^{V}, Z^{V}\right)+Y^{V} \tilde{g}\left(Z^{V}, X^{H}\right)-Z^{V} \tilde{g}\left(X^{H}, Y^{V}\right) \\
& +\tilde{g}\left(Z^{V},\left[X^{H}, Y^{V}\right]\right)+\tilde{g}\left(Y^{V},\left[Z^{V}, X^{H}\right]\right)-\tilde{g}\left(X^{H},\left[Y^{V}, Z^{V}\right]\right) \\
= & X^{H} \tilde{g}\left(Y^{V}, Z^{V}\right)+\tilde{g}\left(Z^{V},\left[X^{H}, Y^{V}\right]\right)+\tilde{g}\left(Y^{V},\left[Z^{V}, X^{H}\right]\right) \\
= & \frac{1}{f} X(f) \tilde{g}\left(Y^{V}, Z^{V}\right)+\tilde{g}\left(\left(\nabla_{X} Y\right)^{V}, Z^{V}\right)+\tilde{g}\left(Y^{V},\left(\nabla_{X} Z\right)^{V}\right) \\
& +\tilde{g}\left(Z^{V},\left(\nabla_{X} Y\right)^{V}\right)-\tilde{g}\left(Y^{V},\left(\nabla_{X} Z\right)^{V}\right) \\
= & 2 \tilde{g}\left(\left(\nabla_{X} Y\right)^{V}, Z^{V}\right)+\frac{1}{f} X(f) \tilde{g}\left(Y^{V}, Z^{V}\right) \\
= & 2 \tilde{g}\left(\left(\nabla_{X} Y\right)^{V}+\frac{1}{2 f} X(f) Y^{V}, Z^{V}\right) .
\end{aligned}
$$

we have:

$$
\widetilde{\nabla}_{X^{H}} Y^{V}=\left(\nabla_{X} Y\right)^{V}+\frac{1}{2 f} X(f) Y^{V}+\frac{f}{2}(R(u, Y) X)^{H} .
$$

The other formulas are obtained by a similar calculation. 


\section{Vertically rescaled Berger-type deformed Sasaki metric and Harmonicity}

4.1. Harmonicity of a vector field $X:(M, g) \longrightarrow(T M, \tilde{g})$.

Lemma 4.1. [10] Let $(M, g)$ be a Riemannian manifold. If $X, Y \in \Im_{0}^{1}(M)$ are vector fields on $M$ and $(x, u) \in T M$ such that $Y_{x}=u$, then we have:

$$
d_{x} Y\left(X_{x}\right)=X_{(x, u)}^{H}+\left(\nabla_{X} Y\right)_{(x, u)}^{V}
$$

Proof. Let $\left(U, x^{i}\right)$ be a local chart on $M$ in $x \in M$ and $\left(\pi^{-1}(U), x^{i}, y^{j}\right)$ be the induced chart on $T M$, if $X_{x}=\left.X^{i}(x) \frac{\partial}{\partial x^{i}}\right|_{x}$ and $Y_{x}=\left.Y^{i}(x) \frac{\partial}{\partial x^{i}}\right|_{x}=u$, then

$$
\begin{aligned}
d_{x} Y\left(X_{x}\right)= & \left.X^{i}(x) \frac{\partial}{\partial x^{i}}\right|_{(x, u)}+\left.X^{i}(x) \frac{\partial Y^{k}}{\partial x^{i}}(x) \frac{\partial}{\partial y^{k}}\right|_{(x, u)} \\
= & \left.X^{i}(x) \frac{\partial}{\partial x^{i}}\right|_{(x, u)}-\left.X^{i}(x) Y^{j}(x) \Gamma_{i j}^{k}(x) \frac{\partial}{\partial y^{k}}\right|_{(x, u)} \\
& +\left.X^{i}(x) Y^{j}(x) \Gamma_{i j}^{k}(x) \frac{\partial}{\partial y^{k}}\right|_{(x, u)}+\left.X^{i}(x) \frac{\partial Y^{k}}{\partial x^{i}}(x) \frac{\partial}{\partial y^{k}}\right|_{(x, u)} \\
= & {\left[X^{i}\left(\frac{\partial}{\partial x^{i}}-y^{j} \Gamma_{i j}^{k}\right) \frac{\partial}{\partial y^{k}}\right]_{(x, u)}+\left[X^{i}\left(\frac{\partial Y^{k}}{\partial x^{i}}+Y^{j} \Gamma_{i j}^{k}\right) \frac{\partial}{\partial y^{k}}\right]_{(x, u)} } \\
= & X_{(x, u)}^{H}+\left[X^{i}\left(\frac{\partial Y^{k}}{\partial x^{i}}+Y^{j} \Gamma_{i j}^{k}\right) \frac{\partial}{\partial x^{k}}\right]_{(x, u)}^{V} \\
= & X_{(x, u)}^{H}+\left(\nabla_{X} Y\right)_{(x, u)}^{V} .
\end{aligned}
$$

Lemma 4.2. Let $\left(M^{2 m}, \varphi, g\right)$ be an almost anti-paraHermitian manifold and $(T M, \tilde{g})$ be its tangent bundle equipped with the vertically rescaled Berger-type deformed Sasaki metric. If $X \in \Im_{0}^{1}(M)$, then the energy density associated to $X$ is given by

$$
e(X)=m+\frac{f}{2} \operatorname{trace}_{g}\left[g(\nabla X, \nabla X)+\delta^{2} g(\nabla X, \varphi X)^{2}\right]
$$

Proof. Let $(x, u) \in T M, X \in \Im_{0}^{1}(M), X_{x}=u$ and $\left\{E_{i}\right\}_{i=\overline{1,2 m}}$ be a local orthonormal frame on $M$, then:

$$
\begin{aligned}
e(X)_{x} & =\frac{1}{2} \operatorname{trace}_{g} \tilde{g}(d X, d X)_{(x, u)} \\
& =\frac{1}{2} \sum_{i=1}^{2 m} \tilde{g}\left(d X\left(E_{i}\right), d X\left(E_{i}\right)\right)_{(x, u)}
\end{aligned}
$$


Using Lemma 4.1, we obtain:

$$
\begin{aligned}
e(X) & =\frac{1}{2} \sum_{i=1}^{2 m} \tilde{g}\left(E_{i}^{H}+\left(\nabla_{E_{i}} X\right)^{V}, E_{i}^{H}+\left(\nabla_{E_{i}} X\right)^{V}\right) \\
& =\frac{1}{2} \sum_{i=1}^{2 m}\left(\tilde{g}\left(E_{i}^{H}, E_{i}^{H}\right)+\tilde{g}\left(\left(\nabla_{E_{i}} X\right)^{V},\left(\nabla_{E_{i}} X\right)^{V}\right)\right) \\
& =\frac{1}{2} \sum_{i=1}^{2 m}\left(g\left(E_{i}, E_{i}\right)+f\left(g\left(\nabla_{E_{i}} X, \nabla_{E_{i}} X\right)+\delta^{2} g\left(\nabla_{E_{i}} X, \varphi X\right)^{2}\right)\right) \\
& =m+\frac{f}{2} \operatorname{trace}_{g}\left(g(\nabla X, \nabla X)+\delta^{2} g(\nabla X, \varphi X)^{2}\right) .
\end{aligned}
$$

Theorem 4.1. Let $\left(M^{2 m}, \varphi, g\right)$ be an anti-paraKähler manifold and $(T M, \tilde{g})$ be its tangent bundle equipped with the vertically rescaled Berger-type deformed Sasaki metric. If $X \in \Im_{0}^{1}(M)$, then the tension field associated to $X$ is given by:

$$
\tau(X)=\left[\operatorname{trace}_{g} A(X)\right]^{H}+\left[\frac{1}{f} \nabla_{\text {grad } f} X+\operatorname{trace}_{g} B(X)\right]^{V},
$$

where $A(X)$ and $B(X)$ are a bilinear maps defined by

$$
\begin{aligned}
& A(X)=f R(X, \nabla X) *-\frac{1}{2}\left(g(\nabla X, \nabla X)+\delta^{2} g(\nabla X, \varphi X)^{2}\right) \operatorname{grad} f, \\
& B(X)=\nabla^{2} X+\frac{\delta^{2}}{\lambda} g(\nabla X, \varphi(\nabla X)) \varphi X,
\end{aligned}
$$

where $\lambda=1+\delta^{2}\|X\|^{2}$ and $\|X\|^{2}=g(X, X)$. In the expression $R(X, \nabla X) *$, the symbol * denotes the third component of the curvature tensor.

Proof. Let $(x, u) \in T M, X \in \Im_{0}^{1}(M), X_{x}=u$ and $\left\{E_{i}\right\}_{i=1,2 m}$ be a local orthonormal frame on $M$ such that $\left(\nabla_{E_{i}}^{M} E_{i}\right)_{x}=0$, then

$$
\begin{aligned}
\tau(X)_{x}= & \sum_{i=1}^{2 m}\left(\nabla_{E_{i}}^{X} d X\left(E_{i}\right)-d X\left(\nabla_{E_{i}}^{M} E_{i}\right)\right)_{x} \\
= & \sum_{i=1}^{2 m}\left(\widetilde{\nabla}_{\left(E_{i}^{H}+\left(\nabla_{E_{i}} X\right)^{V}\right)}\left(E_{i}^{H}+\left(\nabla_{E_{i}} X\right)^{V}\right)\right)_{(x, u)} \\
= & \sum_{i=1}^{2 m}\left(\widetilde{\nabla}_{E_{i}^{H}} E_{i}^{H}+\widetilde{\nabla}_{E_{i}^{H}}\left(\nabla_{E_{i}} X\right)^{V}+\widetilde{\nabla}_{\left(\nabla_{E_{i}} X\right)^{V}}\left(E_{i}\right)^{H}\right. \\
& \left.+\widetilde{\nabla}_{\left(\nabla_{E_{i}} X\right)^{V}}\left(\nabla_{E_{i}} X\right)^{V}\right)_{(x, u)}
\end{aligned}
$$


Using Theorem 3.1, we obtain

$$
\begin{aligned}
\tau(X)= & \sum_{i=1}^{2 m}\left(\left(\nabla_{E_{i}} E_{i}\right)^{H}-\frac{1}{2}\left(R\left(E_{i}, E_{i}\right) X\right)^{V}+\left(\nabla_{E_{i}} \nabla_{E_{i}} X\right)^{V}\right. \\
& +\frac{1}{2 f} E_{i}(f)\left(\nabla_{E_{i}} X\right)^{V}+\frac{f}{2}\left(R\left(X, \nabla_{E_{i}} X\right) E_{i}\right)^{H}+\frac{1}{2 f} E_{i}(f)\left(\nabla_{E_{i}} X\right)^{V} \\
& +\frac{f}{2}\left(R\left(X, \nabla_{E_{i}} X\right) E_{i}\right)^{H}-\frac{1}{2 f} \tilde{g}\left(\left(\nabla_{E_{i}} X\right)^{V},\left(\nabla_{E_{i}} X\right)^{V}\right)(\operatorname{grad} f)^{H} \\
& \left.+\frac{\delta^{2}}{\lambda} g\left(\nabla_{E_{i}} X, \varphi\left(\nabla_{E_{i}} X\right)\right)(\varphi X)^{V}\right) \\
= & \sum_{i=1}^{2 m}\left(f\left(R\left(X, \nabla_{E_{i}} X\right) E_{i}\right)^{H}-\frac{1}{2 f} \tilde{g}\left(\left(\nabla_{E_{i}} X\right)^{V},\left(\nabla_{E_{i}} X\right)^{V}\right)(\operatorname{grad} f)^{H}\right. \\
& \left.+\left(\nabla_{E_{i}} \nabla_{E_{i}} X\right)^{V}+\frac{1}{f} E_{i}(f)\left(\nabla_{E_{i}} X\right)^{V}+\frac{\delta^{2}}{\lambda} g\left(\nabla_{E_{i}} X, \varphi\left(\nabla_{E_{i}} X\right)\right)(\varphi X)^{V}\right) \\
= & \left(\operatorname{trace}_{g}\left(f R(X, \nabla X) *-\frac{1}{2}\left(g(\nabla X, \nabla X)+\delta^{2} g(\nabla X, \varphi X)^{2}\right) \operatorname{grad} f\right)\right)^{H} \\
& +\left(\frac{1}{f} \nabla_{\text {grad }} X+\operatorname{trace}_{g}\left(\nabla^{2} X+\frac{\delta^{2}}{\lambda} g(\nabla X, \varphi(\nabla X)) \varphi X\right)\right)^{V} .
\end{aligned}
$$

Theorem 4.2. Let $\left(M^{2 m}, \varphi, g\right)$ be an anti-paraKähler manifold and $(T M, \tilde{g})$ be its tangent bundle equipped with the vertically rescaled Berger-type deformed Sasaki metric. If $X \in \Im_{0}^{1}(M), X$ is a harmonic vector field if and only if the following conditions are verified

$$
\text { trace }_{g}\left(f R(X, \nabla X) *-\frac{f}{2}\left(g(\nabla X, \nabla X)+\delta^{2} g(\nabla X, \varphi X)^{2}\right) \operatorname{grad} f\right)=0,
$$

and

$$
\frac{1}{f} \nabla_{\text {grad } f} X+\operatorname{trace}_{g}\left(\nabla^{2} X+\frac{\delta^{2}}{\lambda} g(\nabla X, \varphi(\nabla X)) \varphi X\right)=0,
$$

where $\lambda=1+\delta^{2}\|X\|^{2}$ and $\|X\|^{2}=g(X, X)$.

Proof. The statement is a direct consequence of Theorem 4.1.

Corollary 4.1. Let $\left(M^{2 m}, \varphi, g\right)$ be an anti-paraKähler manifold and $(T M, \tilde{g})$ be its tangent bundle equipped with the vertically rescaled Berger-type deformed Sasaki metric. If $X \in \Im_{0}^{1}(M), X$ is a parallel vector field (i.e. $\nabla X=0$ ) then $X$ is harmonic.

Example 4.1. Let $\mathbb{R}^{2}$ be endowed with the structure anti-paraKähler $(\varphi, g)$ defined by

and

$$
g=e^{2 x} d x^{2}+e^{2 y} d y^{2},
$$

$$
\varphi \frac{\partial}{\partial x}=\frac{e^{x}}{e^{y}} \frac{\partial}{\partial y} \quad, \quad \varphi \frac{\partial}{\partial y}=\frac{e^{y}}{e^{x}} \frac{\partial}{\partial x} .
$$

The non-null Christoffel symbols of the Riemannian connection are:

$$
\Gamma_{11}^{1}=\Gamma_{22}^{2}=1
$$


then we have,

$$
\nabla_{\frac{\partial}{\partial x}} \frac{\partial}{\partial x}=\frac{\partial}{\partial x}, \nabla_{\frac{\partial}{\partial x}} \frac{\partial}{\partial y}=\nabla_{\frac{\partial}{\partial y}} \frac{\partial}{\partial x}=0, \nabla_{\frac{\partial}{\partial y}} \frac{\partial}{\partial y}=\frac{\partial}{\partial y},
$$

the vector field $X=\frac{1}{e^{x}} \frac{\partial}{\partial x}+\frac{1}{e^{y}} \frac{\partial}{\partial y}$ is harmnic because $X$ is parallel, indeed,

$$
\begin{aligned}
& \nabla_{\frac{\partial}{\partial x}} X=-\frac{1}{e^{x}} \frac{\partial}{\partial x}+\frac{1}{e^{x}} \nabla_{\frac{\partial}{\partial x}} \frac{\partial}{\partial x}+\frac{1}{e^{y}} \nabla_{\frac{\partial}{\partial x}} \frac{\partial}{\partial y}=0 \\
& \nabla_{\frac{\partial}{\partial \theta}} X=\frac{1}{e^{x}} \nabla_{\frac{\partial}{\partial y}} \frac{\partial}{\partial x}-\frac{1}{e^{y}} \frac{\partial}{\partial y}+\frac{1}{e^{y}} \nabla_{\frac{\partial}{\partial y}} \frac{\partial}{\partial y}=0
\end{aligned}
$$

i.e. $\nabla X=0$, then $X$ is harmonic.

Example 4.2. Let $\mathbb{R}^{2}$ be endowed with the structure anti-paraKähler $(\varphi, g)$ in polar coordinate defined by

$$
g=d r^{2}+r^{2} d \theta^{2}
$$

and

$$
\varphi \frac{\partial}{\partial r}=\sin 2 \theta \frac{\partial}{\partial r}+\frac{1}{r} \cos 2 \theta \frac{\partial}{\partial \theta} \quad, \quad \varphi \frac{\partial}{\partial \theta}=r \cos 2 \theta \frac{\partial}{\partial r}-\sin 2 \theta \frac{\partial}{\partial \theta} .
$$

The non-null Christoffel symbols of the Riemannian connection are:

$$
\Gamma_{12}^{2}=\Gamma_{21}^{2}=\frac{1}{r}, \Gamma_{22}^{1}=-r
$$

then we have,

$$
\nabla_{\frac{\partial}{\partial r}} \frac{\partial}{\partial r}=0, \nabla_{\frac{\partial}{\partial r}} \frac{\partial}{\partial \theta}=\nabla_{\frac{\partial}{\partial \theta}} \frac{\partial}{\partial r}=\frac{1}{r} \frac{\partial}{\partial \theta}, \nabla_{\frac{\partial}{\partial \theta}} \frac{\partial}{\partial \theta}=-r \frac{\partial}{\partial r},
$$

the vector field $X=\sin \theta \frac{\partial}{\partial r}+\frac{1}{r} \cos \theta \frac{\partial}{\partial \theta}$ is harmnic because $X$ is parallel, indeed,

$$
\begin{gathered}
\nabla_{\frac{\partial}{\partial r}} X=\sin \theta \nabla_{\frac{\partial}{\partial r}} \frac{\partial}{\partial r}-\frac{1}{r^{2}} \cos \theta \frac{\partial}{\partial \theta}+\frac{1}{r} \cos \theta \nabla_{\frac{\partial}{\partial r}} \frac{\partial}{\partial \theta}=0 \\
\nabla_{\frac{\partial}{\partial \theta}} X=\cos \theta \frac{\partial}{\partial r}+\sin \theta \nabla_{\frac{\partial}{\partial \theta}} \frac{\partial}{\partial r}-\frac{1}{r} \sin \theta \frac{\partial}{\partial \theta}+\frac{1}{r} \cos \theta \nabla_{\frac{\partial}{\partial \theta}} \frac{\partial}{\partial \theta}=0,
\end{gathered}
$$

i.e. $\nabla X=0$, then $X$ is harmonic.

Theorem 4.3. Let $\left(M^{2 m}, \varphi, g\right)$ be an anti-paraKähler compact manifold and $(T M, \tilde{g})$ be its tangent bundle equipped with the vertically rescaled Berger-type deformed Sasaki metric. If $X \in \Im_{0}^{1}(M), X$ is a harmonic vector field if and only if $X$ is parallel (i.e. $\nabla X=0)$.

Proof. If $X$ is parallel, from Corollary 4.1, we deduce that $X$ is harmonic vector field.

Inversely, let $X_{t}$ be a compactly supported variation of $X$ defined by:

$$
\begin{aligned}
\mathbb{R} \times M & \longrightarrow T_{x} M \\
(t, x) & \longmapsto X_{t}(x)=(1+t) X_{x}
\end{aligned}
$$


From lemma 4.2 we have:

$$
\begin{aligned}
e\left(X_{t}\right)= & m+\frac{(1+t)^{2}}{2} f \operatorname{trace}_{g} g(\nabla X, \nabla X)+\frac{(1+t)^{4}}{2} f \delta^{2} \text { trace }_{g} g(\nabla X, \varphi X)^{2}, \\
E\left(X_{t}\right)= & m \operatorname{Vol}(M)+\frac{(1+t)^{2}}{2} \int_{M} f \operatorname{trace}_{g} g(\nabla X, \nabla X) d v_{g} \\
& +\frac{(1+t)^{4}}{2} \delta^{2} \int_{M} \text { trace }_{g} g(\nabla X, \varphi X)^{2} d v_{g} .
\end{aligned}
$$

If $X$ is a critical point of the energy functional, then we have :

$$
\begin{aligned}
0= & \left.\frac{\partial}{\partial t} E\left(X_{t}\right)\right|_{t=0} \\
= & \frac{\partial}{\partial t}\left[m \operatorname{Vol}(M)+\frac{(1+t)^{2}}{2} \int_{M} f \operatorname{trace}_{g} g(\nabla X, \nabla X) d v_{g}\right]_{t=0} \\
& +\frac{\partial}{\partial t}\left[\frac{(1+t)^{4}}{2} \delta^{2} \int_{M} f \operatorname{trace}_{g} g(\nabla X, \varphi X)^{2} d v_{g}\right]_{t=0} \\
= & \int_{M} f \operatorname{trace}_{g} g(\nabla X, \nabla X) d v_{g}+2 \delta^{2} \int_{M} f \operatorname{trace}_{g} g(\nabla X, \varphi X)^{2} d v_{g} \\
= & \int_{M} \text { frace }_{g}\left[g(\nabla X, \nabla X)+2 \delta^{2} g(\nabla X, \varphi X)^{2}\right] d v_{g}
\end{aligned}
$$

which gives

$$
g(\nabla X, \nabla X)+2 \delta^{2} g(\nabla X, \varphi X)^{2}=0
$$

hence $\nabla X=0$.

Remark 4.1. In general, using Corollary 4.1 and Theorem 4.3, we can construct many examples for a harmonic vector fields.

\subsection{Harmonicity of the map $\sigma:(M, g) \longrightarrow(T N, \tilde{h})$.}

Lemma 4.3. Let $\phi:(M, g) \rightarrow(N, h)$ be a smooth map between Riemannian manifolds and let

$$
\begin{aligned}
\sigma: M & \longrightarrow T N \\
x & \longmapsto(\phi(x), v)
\end{aligned}
$$

be a smooth map such that $\phi=\pi_{N} \circ \sigma$, where $v \in T_{\phi(x)} N$ and $\pi_{N}: T N \rightarrow N$ is the canonical projection, then

$$
d \sigma(X)=(d \phi(X))^{H}+\left(\nabla_{X}^{\phi} \sigma\right)^{V},
$$

for all $X \in \Im_{0}^{1}(M)$, where $\nabla^{\phi}$ is the pull-back connection on the pull-back bundle $\phi^{-1} T N$.

Proof. Let $x \in M, X \in \Im_{0}^{1}(M)$ and $Y \in \Im_{0}^{1}(N)$ such that $Y_{\phi(x)}=v \in T_{\phi(x)} N$. Using Lemma 4.1, we obtain:

$$
\begin{aligned}
d_{x} \sigma\left(X_{x}\right) & =d_{x}(Y \circ \phi)\left(X_{x}\right) \\
& =d_{\phi(x)} Y\left(d_{x} \phi\left(X_{x}\right)\right) \\
& =(d \phi(X))_{(\phi(x), v)}^{H}+\left(\nabla_{d \phi(X)} Y\right)_{(\phi(x), v)}^{V} \\
& =(d \phi(X))_{(\phi(x), v)}^{H}+\left(\nabla_{X}^{\phi} \sigma\right)_{(\phi(x), v)}^{V} .
\end{aligned}
$$


Theorem 4.4. Let $\left(M^{m}, g\right)$ be a Riemannian manifold, $\left(N^{2 n}, \varphi, h\right)$ be an antiparaKähler manifold and let $(T N, \tilde{h})$ be the tangent bundle of $N$ equipped with vertically rescaled Berger-type deformed Sasaki metric. Let $\phi: M \rightarrow N$ be a smooth map and

$$
\begin{aligned}
\sigma: M & \longrightarrow T N \\
x & \longmapsto(\phi(x), v)
\end{aligned}
$$

be a smooth map such that $\phi=\pi_{N} \circ \sigma$ and $v \in T_{\phi(x)} N$. The tension field of $\sigma$ is given by

$$
\begin{aligned}
\tau(\sigma)= & \left(\tau(\phi)+\operatorname{trace}_{g}\left(f R^{N}\left(\sigma, \nabla^{\phi} \sigma\right) d \phi\right.\right. \\
& \left.\left.-\frac{1}{2}\left(h\left(\nabla^{\phi} \sigma, \nabla^{\phi} \sigma\right)+\delta^{2} h\left(\nabla^{\phi} \sigma, \varphi \sigma\right)^{2}\right) \operatorname{grad} f\right)\right)^{H} \\
& +\left(\text { trace }_{g}\left(\left(\nabla^{\phi}\right)^{2} \sigma+\frac{1}{f} h(\operatorname{grad} f, d \phi) \nabla^{\phi} \sigma+\frac{\delta^{2}}{\lambda} h\left(\nabla^{\phi} \sigma, \varphi \nabla^{\phi} \sigma\right) \varphi \sigma\right)\right)^{V},
\end{aligned}
$$

where $\lambda=1+\delta^{2}\|\sigma\|^{2},\|\sigma\|^{2}=h(\sigma, \sigma)$ and $\nabla^{\phi}$ is the pull-back connection.

Proof. Let $x \in M$ and $\left\{E_{i}\right\}_{i=\overline{1, m}}$ be a local orthonormal frame on $M$ such that $\left(\nabla_{E_{i}}^{M} E_{i}\right)_{x}=0$ and $\sigma(x)=(\phi(x), v), v \in T_{\phi(x)} N$. Using Lemma 4.3, we have

$$
\begin{aligned}
\tau(\sigma)_{x}= & \sum_{i=1}^{m}\left(\left(\nabla_{E_{i}}^{\sigma} d \sigma\left(E_{i}\right)\right)_{x}-d \sigma\left(\nabla_{E_{i}}^{M} E_{i}\right)_{x}\right) \\
= & \sum_{i=1}^{m}\left(\nabla_{d \sigma\left(E_{i}\right)}^{T N} d \sigma\left(E_{i}\right)\right)_{(\phi(x), v)} \\
= & \sum_{i=1}^{m}\left(\nabla_{\left(\left(d \phi\left(E_{i}\right)\right)^{H}+\left(\nabla_{E_{i}}^{\phi} \sigma\right)^{V}\right)}^{T N}\left(\left(d \phi\left(E_{i}\right)\right)^{H}+\left(\nabla_{E_{i}}^{\phi} \sigma\right)^{V}\right)\right)_{(\phi(x), v)} \\
= & \sum_{i=1}^{m}\left(\nabla_{\left(d \phi\left(E_{i}\right)\right)^{H}}^{T N}\left(d \phi\left(E_{i}\right)\right)^{H}+\nabla_{\left(d \phi\left(E_{i}\right)\right)^{H}}^{T N}\left(\nabla_{E_{i}}^{\phi} \sigma\right)^{V}+\nabla_{\left(\nabla_{E_{i}}^{\phi} \sigma\right)^{V}}^{T N}\left(d \phi\left(E_{i}\right)\right)^{H}\right. \\
& \left.+\nabla_{\left(\nabla_{E_{i}}^{\phi} \sigma\right)^{V}}^{T N}\left(\nabla_{E_{i}}^{\phi} \sigma\right)^{V}\right)_{(\phi(x), v)}
\end{aligned}
$$

From the Theorem 3.1, we obtain:

$$
\begin{aligned}
\tau(\sigma)= & \sum_{i=1}^{m}\left(\left(\nabla_{d \phi\left(E_{i}\right)}^{N} d \phi\left(E_{i}\right)\right)^{H}-\frac{1}{2}\left(R^{N}\left(d \phi\left(E_{i}\right), d \phi\left(E_{i}\right)\right) \sigma\right)^{V}+\left(\nabla_{d \phi\left(E_{i}\right)}^{N} \nabla_{E_{i}}^{\phi} \sigma\right)^{V}\right. \\
& +\frac{1}{2 f} d \phi\left(E_{i}\right)(f)\left(\nabla_{E_{i}}^{\phi} \sigma\right)^{V}+\frac{f}{2}\left(R^{N}\left(\sigma, \nabla_{E_{i}}^{\phi} \sigma\right) d \phi\left(E_{i}\right)\right)^{H} \\
& +\frac{1}{2 f} d \phi\left(E_{i}\right)(f)\left(\nabla_{E_{i}}^{\phi} \sigma\right)^{V}+\frac{f}{2}\left(R^{N}\left(\sigma, \nabla_{E_{i}}^{\phi} \sigma\right) d \phi\left(E_{i}\right)\right)^{H} \\
& \left.-\frac{1}{2 f} \tilde{h}\left(\left(\nabla_{E_{i}}^{\phi} \sigma\right)^{V},\left(\nabla_{E_{i}}^{\phi} \sigma\right)^{V}\right)(\operatorname{grad} f)^{H}+\frac{\delta^{2}}{\lambda} h\left(\nabla_{E_{i}}^{\phi} \sigma, \varphi \nabla_{E_{i}}^{\phi} \sigma\right)(\varphi \sigma)^{V}\right)
\end{aligned}
$$




$$
\begin{aligned}
= & \sum_{i=1}^{m}\left(\left(\nabla_{E_{i}}^{\phi} d \phi\left(E_{i}\right)\right)^{H}+f\left(R^{N}\left(\sigma, \nabla_{E_{i}}^{\phi} \sigma\right) d \phi\left(E_{i}\right)\right)^{H}\right. \\
& -\frac{1}{2}\left(h\left(\nabla_{E_{i}}^{\phi} \sigma, \nabla_{E_{i}}^{\phi} \sigma\right)+\delta^{2} h\left(\nabla_{E_{i}}^{\phi} \sigma, \varphi \sigma\right)^{2}\right)(\operatorname{grad} f)^{H}+\left(\nabla_{E_{i}}^{\phi} \nabla_{E_{i}}^{\phi} \sigma\right)^{V} \\
& \left.+\frac{1}{f} h\left(\operatorname{grad} f, d \phi\left(E_{i}\right)\right)\left(\nabla_{E_{i}}^{\phi} \sigma\right)^{V}+\frac{\delta^{2}}{\lambda} h\left(\nabla_{E_{i}}^{\phi} \sigma, \varphi \nabla_{E_{i}}^{\phi} \sigma\right)(\varphi \sigma)^{V}\right) .
\end{aligned}
$$

Therefore we get

$$
\begin{aligned}
= & \left(\tau(\phi)+\operatorname{trace}_{g}\left(f R^{N}\left(\sigma, \nabla^{\phi} \sigma\right) d \phi\right.\right. \\
& \left.\left.-\frac{1}{2}\left(h\left(\nabla^{\phi} \sigma, \nabla^{\phi} \sigma\right)+\delta^{2} h\left(\nabla^{\phi} \sigma, \varphi \sigma\right)^{2}\right) \operatorname{grad} f\right)\right)^{H} \\
& +\left(\text { trace }_{g}\left(\left(\nabla^{\phi}\right)^{2} \sigma+\frac{1}{f} h(\operatorname{grad} f, d \phi) \nabla^{\phi} \sigma+\frac{\delta^{2}}{\lambda} h\left(\nabla^{\phi} \sigma, \varphi \nabla^{\phi} \sigma\right) \varphi \sigma\right)\right)^{V} .
\end{aligned}
$$

From Theorem 4.4 we obtain.

Theorem 4.5. Let $\left(M^{m}, g\right)$ be a Riemannian manifold, $\left(N^{2 n}, \varphi, h\right)$ be an antiparaKähler manifold and let $(T N, \tilde{h})$ be the tangent bundle of $N$ equipped with vertically rescaled Berger-type deformed Sasaki metric. Let $\phi: M \rightarrow N$ be a smooth map and

$$
\begin{aligned}
\sigma: M & \longrightarrow T N \\
x & \longmapsto(\phi(x), v)
\end{aligned}
$$

be a smooth map such that $\phi=\pi_{N} \circ \sigma$ and $v \in T_{\phi(x)} N$, then $\sigma$ is a harmonic if and only if the following conditions are verified

$$
\tau(\phi)=\operatorname{trace}_{g}\left(\frac{1}{2}\left(h\left(\nabla^{\phi} \sigma, \nabla^{\phi} \sigma\right)+\delta^{2} h\left(\nabla^{\phi} \sigma, \varphi \sigma\right)^{2}\right) \operatorname{grad} f-f R^{N}\left(\sigma, \nabla^{\phi} \sigma\right) d \phi\right),
$$

and

$$
\text { trace }_{g}\left(\left(\nabla^{\phi}\right)^{2} \sigma+\frac{1}{f} h(\operatorname{grad} f, d \phi) \nabla^{\phi} \sigma+\frac{\delta^{2}}{\lambda} h\left(\nabla^{\phi} \sigma, \varphi \nabla^{\phi} \sigma\right) \varphi \sigma\right)=0 .
$$

\subsection{Harmonicity of the map $\Phi:(T M, \tilde{g}) \longrightarrow(N, h)$.}

Lemma 4.4. Let $\left(M^{2 m}, \varphi, g\right)$ be an anti-paraKähler manifold and $(T M, \tilde{g})$ be its tangent bundle equipped with the vertically rescaled Berger-type deformed Sasaki metric. The tension field of the canonical projection $\pi:(T M, \tilde{g}) \longrightarrow(M, g)$ is given by

$$
\tau(\pi)=\frac{m}{f}(\operatorname{grad} f) \circ \pi .
$$

Proof. Let $(x, u) \in T M$ and $\left\{E_{i}\right\}_{i=\overline{1,2 m}}$ be a local orthonormal frame on $M$ such that $E_{1}=\frac{u}{\|u\|}$, In this case, the set $\left\{F_{a}\right\}_{a=\overline{1,4 m}}$ which is defined as below, is a local orthonormal frame on $T M$.

$$
F_{i}=E_{i}^{H}, F_{2 m+1}=\frac{1}{\sqrt{f \lambda}}\left(\varphi E_{1}\right)^{V}, F_{2 m+j}=\frac{1}{\sqrt{f}}\left(\varphi E_{j}\right)^{V}, i=\overline{1,2 m}, j=\overline{2,2 m} .
$$




$$
\begin{aligned}
\tau(\pi)= & \operatorname{trace}_{\tilde{g}} \nabla d \pi \\
= & \sum_{i=1}^{2 m}\left(\nabla_{F_{i}}^{\pi} d \pi\left(F_{i}\right)-d \pi\left(\nabla_{F_{i}}^{T M} F_{i}\right)\right)+\nabla_{F_{2 m+1}}^{\pi} d \pi\left(F_{2 m+1}\right) \\
& -d \pi\left(\nabla_{F_{2 m+1}}^{T M} F_{2 m+1}\right)+\sum_{j=2}^{2 m}\left(\nabla_{F_{2 m+j}}^{\pi} d \pi\left(F_{2 m+j}\right)-d \pi\left(\nabla_{F_{2 m+j}}^{T M} F_{2 m+j}\right)\right)
\end{aligned}
$$

as $d \pi\left(X^{V}\right)=0$ and $d \pi\left(X^{H}\right)=X \circ \pi$ for any $X \in \Im_{0}^{1}(M)$ then

$$
\begin{aligned}
\tau(\pi)= & \sum_{i=1}^{2 m}\left(\nabla_{d \pi\left(E_{i}^{H}\right)}^{T M} d \pi\left(E_{i}^{H}\right)-d \pi\left(\left(\nabla_{E_{i}}^{M} E_{i}\right)^{H}-\frac{1}{2}\left(R\left(E_{i}, E_{i}\right) u\right)^{V}\right)\right) \\
& -\frac{1}{\sqrt{f \lambda}} d \pi\left(\left(\varphi E_{1}\right)^{V}\left(\frac{1}{\sqrt{f \lambda}}\right)\left(\varphi E_{1}\right)^{V}+\frac{1}{\sqrt{f \lambda}} \nabla_{\left(\varphi E_{1}\right)^{V}}^{T M}\left(\varphi E_{1}\right)^{V}\right) \\
& -\sum_{j=2}^{2 m}\left(\frac{1}{\sqrt{f}} d \pi\left(\left(\varphi E_{j}\right)^{V}\left(\frac{1}{\sqrt{f}}\right)\left(\varphi E_{j}\right)^{V}+\frac{1}{\sqrt{f}} \nabla_{\left(\varphi E_{j}\right)^{V}}^{T M}\left(\varphi E_{j}\right)^{V}\right)\right) \\
= & \sum_{i=1}^{2 m}\left(\nabla_{\left(E_{i} \circ \pi\right)}^{T M}\left(E_{i} \circ \pi\right)-\left(\nabla_{E_{i}}^{M} E_{i}\right) \circ \pi\right) \\
& +\frac{1}{f \lambda} \frac{1}{2 f} \tilde{g}\left(\left(\varphi E_{1}\right)^{V},\left(\varphi E_{1}\right)^{V}\right) d \pi(\operatorname{grad} f)^{H} \\
& +\sum_{j=2}^{2 m} \frac{1}{f \lambda} \frac{1}{2 f} \tilde{g}\left(\left(\varphi E_{j}\right)^{V},\left(\varphi E_{j}\right)^{V}\right) d \pi(\operatorname{grad} f)^{H} \\
= & \frac{1}{2 f \lambda}\left(g\left(\varphi E_{1}, \varphi E_{1}\right)+\delta^{2} g\left(\varphi E_{1}, \varphi u\right)^{2}\right)(\operatorname{grad} f) \circ \pi \\
& +\sum_{j=2}^{2 m} \frac{1}{2 f}\left(g\left(\varphi E_{j}, \varphi E_{j}\right)+\delta^{2} g\left(\varphi E_{j}, \varphi u\right)^{2}\right)(\operatorname{grad} f) \circ \pi \\
= & \frac{1}{2 f}(\operatorname{grad} f) \circ \pi+\frac{2 m-1}{2 f}(\operatorname{grad} f) \circ \pi \\
= & \frac{m}{f}(\operatorname{grad} f) \circ \pi .
\end{aligned}
$$

Theorem 4.6. Let $\left(M^{2 m}, \varphi, g\right)$ be an anti-paraKähler manifold, $\left(N^{n}, h\right)$ be a Riemannian manifold and let $(T M, \tilde{g})$ be the tangent bundle of $M$ equipped with vertically rescaled Berger-type deformed Sasaki metric. Let $\phi:(M, g) \longrightarrow(N, h)$ be a smooth map. The tension field of the map

$$
\begin{aligned}
\Phi:(T M, \tilde{g}) & \longrightarrow(N, h) \\
(x, u) & \longmapsto \phi(x)
\end{aligned}
$$

is given by

$$
\tau(\Phi)=\left(\tau(\phi)+\frac{m}{f} d \phi(\operatorname{grad} f)\right) \circ \pi
$$


Proof. Let $(x, u) \in T M$ and $\left\{E_{i}\right\}_{i=\overline{1,2 m}}$ be a local orthonormal frame on $M$ such that $E_{1}=\frac{u}{\|u\|}$ and $\left\{F_{a}\right\}_{a=\overline{1,4 m}}$ be a local orthonormal frame on $T M$ defined by (4.5). Since $\Phi$ is written in the form $\Phi=\phi \circ \pi$, we have

$$
\begin{aligned}
\tau(\Phi) & =\tau(\phi \circ \pi) \\
& =d \phi(\tau(\pi))+\operatorname{trace}_{\tilde{g}} \nabla d \phi(d \pi, d \pi) \\
\operatorname{trace}_{\tilde{g}} \nabla d \phi(d \pi, d \pi)= & \sum_{i=1}^{2 m}\left(\nabla_{d \pi\left(F_{i}\right)}^{\phi} d \phi\left(d \pi\left(F_{i}\right)\right)-d \phi\left(\nabla_{d \pi\left(F_{i}\right)}^{M} d \pi\left(F_{i}\right)\right)\right) \\
& +\nabla_{d \pi\left(F_{2 m+1}\right)}^{\phi} d \phi\left(d \pi\left(F_{2 m+1}\right)\right)-d \phi\left(\nabla_{d \pi\left(F_{2 m+1}\right)}^{M} d \pi\left(F_{2 m+1}\right)\right) \\
& +\sum_{j=2}^{m}\left(\nabla_{d \pi\left(F_{2 m+j}\right)}^{\phi} d \phi\left(d \pi\left(F_{2 m+j}\right)\right)\right. \\
& \left.-d \phi\left(\nabla_{d \pi\left(F_{2 m+j}\right)}^{M} d \pi\left(F_{2 m+j}\right)\right)\right) \\
= & \sum_{i=1}^{2 m}\left(\nabla_{E_{i}}^{\phi} d \phi\left(E_{i}\right)-d \phi\left(\nabla_{E_{i}}^{M} E_{i}\right)\right) \circ \pi \\
= & \tau(\phi) \circ \pi,
\end{aligned}
$$

Using Lemma 4.4, we obtain:

$$
\begin{aligned}
\tau(\Phi) & =\tau(\phi) \circ \pi+d \phi\left(\frac{m}{f}(\operatorname{grad} f) \circ \pi\right) \\
& =\left[\tau(\phi)+\frac{m}{f} d \phi(\operatorname{grad} f)\right] \circ \pi .
\end{aligned}
$$

Theorem 4.7. Let $\left(M^{2 m}, \varphi, g\right)$ be an anti-paraKähler manifold, $\left(N^{n}, h\right)$ be a Riemannian manifold and let $(T M, \tilde{g})$ be the tangent bundle of $M$ equipped with vertically rescaled Berger-type deformed Sasaki metric. Let $\phi:(M, g) \longrightarrow(N, h)$ a smooth map. The map

$$
\begin{aligned}
\Phi:(T M, \tilde{g}) & \longrightarrow(N, h) \\
(x, u) & \longmapsto \phi(x)
\end{aligned}
$$

is a harmonic if and only if

$$
\tau(\phi)=-\frac{m}{f} d \phi(\operatorname{grad} f) \circ \pi .
$$

Acknowledgements. The author expresses his gratitude to the referee for his valuable comments and suggestions towards the improvement of the paper. The author would also like to thank Dr. Nour Elhouda DJAA, University of Relizane for her helpful suggestions and valuable comments.

This research was supported by the National Algerian P.R.F.U. project. 


\section{References}

[1] M.T.K. Abbassi, M.Sarih, On Natural Metrics on Tangent Bundles of Riemannian Manifolds, Arch. Math. 41 (2005), 71-92.

[2] M. Altunbas, R. Simsek, A. Gezer, A Study Concerning Berger type deformed Sasaki Metric on the Tangent Bundle, Zh. Mat. Fiz. Anal.Geom. 15 (2019), no. 4, 435-447.

[3] V. Cruceanu, P. Fortuny, P.M. Gadea, A survey on paracomplex geometry, Rocky Mountain J. Math. 26 (1996), no. 1, 83-115.

[4] H.M. Dida, F. Hathout, A. Azzouz, On the geometry of the tangent bundle with vertical rescaled metric, Commun. Fac. Sci. Univ. Ank. Ser. A1 Math. Stat. 68 (2019), no. 1, 222-235.

[5] P. Dombrowski, On the Geometry of the tangent bundle, J. Reine Angew. Math. 210 (1962), 73-88.

[6] J. Ells, L. Lemaire, Another report on harmonic maps, Bull. London Math. Soc. 20 (1988), no. 5, 385-524.

[7] J. Eells, J.H. Sampson, Harmonic mappings of Riemannian manifolds, Amer.J. Math. 86 (1964), 109-160.

[8] S. Gudmundsson, E. Kappos, On the geometry of the tangent bundle with the Cheeger-Gromoll metric, Tokyo J. Math. 25 (2002), no. 1, 75-83.

[9] T. Ishihara, Harmonic sections of tangent bundles, J.Math. Tokushima Univ. 13 (1979), 23-27.

[10] F. Latti, M. Djaa, A. Zagane, Mus-Sasaki metric and harmonicity, Math. Sci. Appl. E-Notes 6 (2018), no. 1, 29-36.

[11] E. Musso, F. Tricerri, Riemannian metrics on tangent bundles, Ann. Mat. Pura. Appl. 150(4) (1988), no. 4, 1-19.

[12] V. Opriou, Harmonic Maps Between tangent bundles, Rend. Semin. Mat. Univ. Politec. Torino 47 (1989), no, 1, 47-55.

[13] A.A. Salimov, A. Gezer, K. Akbulut, Geodesics of Sasakian metrics on tensor bundles, Mediterr. J. Math. 6 (2009), no, 2, 135-147.

[14] A.A. Salimov, M. Iscan, F. Etayo, Para-holomorphic B-manifold and its properties, Topology Appl. 154 (2007), no, 4, 925-933.

[15] A.A. Salimov, S. Kazimova, Geodesics of the Cheeger-Gromoll metric, Turk J Math 33 (2009), 99-105.

[16] S. Sasaki, On the differential geometry of tangent bundles of Riemannian manifolds II, Tokyo J. Math. 14 (1962), 146-155.

[17] M. Sekizawa, Curvatures of tangent bundles with Cheeger-Gromoll metric, Tokyo J. Math. 14 (1991), no. 2, 407-417.

[18] K. Yano, M. Ako, On certain operators associated with tensor field, Kodai Math. Sem. Rep. 20 (1968), 414-436.

[19] K. Yano, S. Ishihara, Tangent and cotangent bundles, Marcel Dekker. INC, New York, 1973.

Abderrahim Zagane

Department of Mathematics, University of Relizane, 48000, Relizane, Algeria E-mail address: Zaganeabr2018@gmail.com

Received: February 17, 2021; Revised: July 4, 2021; Accepted: July 8, 2021 